\title{
A Social Bonds Integration Approach for Crowd Panic Simulation
}

\author{
Imene Bouderbal \\ Ecole Nationale Preparatoire aux Etudes \\ d'Ingeniorat, Rouiba, Algiers, Algeria \\ Email: imene.bouderbal@yahoo.com
}

\author{
Abdenour Amamra \\ Ecole Militaire Polytechnique, \\ Bordj El-Bahri BP 17, Algiers, Algeria \\ Email: amamra.abdenour@gmail.com
}

\begin{abstract}
Crowd panic has incurred massive injuries and deaths throughout history; thus understanding it is particularly important in order to save human lives. Recently, numerous simulation methods have been contributed in order to provide insight into the design of evacuation planning strategies. In this paper, we integrate a social structure to the crowd mobility model for the purpose of investigating the influence of social bonds on collective behavior during panic. A macroscopic crowd panic model based on social science theories was integrated as an internal module to the microscopic mobility model. The resulting framework is tunable and permits the implementation of several panic scenarios. It is also designed to run in different situations for a better comprehension of panic-related phenomena. The results demonstrate the smoothness of our crowd flow model and the realism of evacuation during panic.
\end{abstract}

Index Terms-Panic modeling, Crowd simulation, Human behavior in panic, Evacuation disaster.

\section{INTRODUCTION}

$\mathbf{T}$ HROUGHOUT history, mankind has experienced several disasters and accidents caused by panic in overcrowded spaces (Fig 1a). Hence, simulating crowd with panic has an important value in emergency planning for architectures characterized by a high-density crowd. Special attention should be dedicated to a deeper understanding of the nature of panic during the process of escaping hazardous situations within buildings and public spaces [1]. On the other hand, a clearer understanding of the social interactions in difficult situations would give more insights on how we could prevent casualties beforehand by adapting the design of buildings to the potential course of events during an evacuation situation. The understanding of such behavior brings a significant architectural added value, which in turn would result in safer and more reliable buildings.

The prediction of the birth of collective panic and the possible actions that might cause material damage and human losses can be difficult. Modeling and simulation constitute a solution to tackle such a problem. These tools allow us to generate virtual environments including both human agents and buildings, an evacuation scenario can then be run under some assumptions on a given emergency scheme.

Our objective is the modeling of panic propagation process and its consequences on human behavior while taking into account the social aspect of the crowd. To this end, the remainder of the paper is structured as follows: in Section
2 we describe the main related works that were contributed in the field of crowd simulation. in Section 3 we present a brief description of crowd behavior during panic situations. Section 4 is the core of our work, where we propose a sociallyaware panic model. In Section 5, we present the results of our contribution and we discuss their impact compared to alternative methods. Finally, we conclude the paper, and we trace the potential perspectives that can extend the current work.

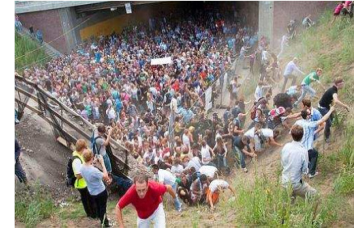

(a)

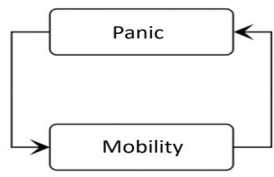

(b)
Fig. 1: (a) Crowd in panic, (b) Systemic loop of panic.

\section{RELATED WORKS}

Crowd simulation models have roots in different domains such as entertainment, security, urbanism, architecture, management, etc. Since we are interested in panic modeling, we will present the main works that have permitted the modeling of panic evolution phenomenon and its effects on the crowd as well as those which gave consistent results with empirical observations. Indeed, we will focus on conceptual modeling rather than the available software tools. After analyzing collective behaviors literature, three main theoretical approaches were found.

The first one, called Contagion Theory [2, 3], it states that an individual in a crowd loses their conscious personality and obeys to all suggestions from the crowd influent members. Works belonging to this category incorporate the concept of social forces, where the model allows a mixture of sociopsychological and physical forces influencing the behaviors within a crowd [4]. Several models based on this approach have been developed so far, the latter proposes an adaptation and hybridization of the model of social forces with other models [5]. These models can reproduce certain phenomena observed in real crowds during panic movements, such as crowd oscillation in a narrow passage, arch formation in front 
of an exit, lane formation, etc. However, they neglect the description of panic evolution phenomenon and reproduce only some of its aspects. Moreover, the social structure of the crowd is neglected and the evolution of the emotional state of individuals is not taken into account, which impinges on the realism.

The second approach is based on the Emergent Norm Theory [6], which considers that the collective behavior is an outcome of the interactions among individuals, which are able to evaluate the information they receive and to decide on its usage at the present situation. In fact, agents assign positive or negative values to the information, which leads to the development of an interactive cognition. This approach analyzes the agent's micro-properties that help in the social system formation. It also analyzes the behavior patterns at a group level. An example of works based on this approach can be found in [7], where the authors describe an individual behavior model for agents. Although there is no explicit way of interaction or communication, each agent is aware of itself and their peers.

The last approach is a variant of the second one, commonly called Structuralism, it inverts the formula and emphasizes the social structure studies and their impact on individuals. It is a macro-to-micro approach since it considers that the changes are triggered by the crowd (macro) to the individuals (micro). Social science research such as [8] embraced the structural theory approach. One of the works that had implemented the macro-to-micro approach for panic crowd situations is [9], where the authors utilized system dynamics and consider that panic is a domino effect. In fact, the movements of individuals result from two complementary models that interact and influence each other mutually, namely the mobility model and the panic model (Fig 1b).

These models are more informative than those based on alternative approaches. In fact, they allow us to describe the beginning and the evolution of panic, thereby to adapt the behaviors to the individual state. However, the solutions that were found model sudden transitions between different states of human emotions. In fact, before an individual behaves in a non-rational fashion, they go through a stage called Limited Rationality and can remain in it or calm down without reaching the state of panic. Also, these models do not take into account the social structure of the crowd.

\section{CROWD PANIC}

Understanding how crowds behave during critical situations has long been necessary for emergency response and management. In fact, most of the normal behavior vanishes when pedestrians face an emergency situation (it does not always have to be an emergency situation, however, similar actions can be observed for example among crowds trying to get the best seats at a concert or consumers running for sales) and non-adaptive crowd behaviors appear. These behaviors are recognized to be responsible for the death and injury of most victims in crowd disasters. Non-adaptive crowd behaviors refer to the destructive actions that a crowd may experience in emergency situations, such as stampede, pushing, knocking and trampling on others [1]. Generally, these behaviors are the result of a dangerous psycho-sociological phenomenon, which is panic.

Panic is a phenomenon generally studied in psychology and human science and often identified by its consequences. It is triggered whenever a situation of tension worsens, slips or escapes from human control. Panic is defined as an intense fear triggered by the occurrence of a real or imaginary danger felt simultaneously by all individuals in a group, a crowd or a population, characterized by the regression of mentalities to an archaic and gregarious level, leading to primitive reactions of hopeless jumps, indiscriminate agitation of violence or collective suicide [5]. Nevertheless, discrepancies exist between the definition of triggering and propagation processes. This difference gives rise to different social theories [1]. The latter differs in the definition of the relationship between disasters and panic phenomenon, and the type of behavior in a state of panic. Despite the differences, these approaches come together on two important points:

- Panic is a feeling of extreme fear that invades the pedestrian following the perception of possible danger;

- Social interaction in a crowd promotes the spread of panic.

\section{PRoposed MODEL}

In order to ensure the safety of people and reduce the impact of panic caused by disasters, it is necessary to understand and model this dangerous phenomenon, its propagation process, and its consequences. Panic model presented hereinafter is based on system dynamics. It has been integrated into a mobility model for crowd evacuation simulation during catastrophes. Besides, it uses the same environment and pedestrian modeling (Fig 1b). The proposed mobility model is microscopic, whereas the proposed panic model is macroscopic (Fig 2). Thereby, panic model permits the study of the evolution of panic phenomenon at a different level of granularity than that adopted for the study of mobility. Many types of relations coexist between the individuals of a crowd (family bonds, friendships, professional relations, etc.). Behaviors and decisions made by these individuals depend strongly on the social relations $[10,11]$. We added a social model that describes the social structure of the crowd and its evolution during the emergency in order to study their effects on pedestrians mobility and the propagation of panic.

\section{A. Social structure of the crowd}

In order to build an innovative solution for crowd modeling, we chose to integrate an important aspect into our mobility model which is the social structure of the crowd. This latter could be integrated by using a social model [2]. The one we adopted in our work is Small World because its generated graphs are the closest to the real representation of contacts between individuals and groups and their evolution in space and time. These graphs allow us to predict the evolution of the epidemic and by analogy the evolution of panic. 
We used a new model for generating small world graphs. Transformations and constructions that it orchestrates follow sociological behaviors observed and described in the literature. The generation process provided by this model is divided into three phases, each permits to accomplish a different aspect of links evolution in the crowd.

1) Initialization: The model we used is configurable. The generative process behind it permits to create a simple, connected and undirected graph $G=(V ; E)$, of $|V|$ nodes and $|E|$ edges.

2) Creating nodes (inserting agents into the simulated environment): The first step allows the addition of new individuals in the social structure of the crowd. In fact, every time an agent is added to the simulation environment, they join the social structure. The graph thus obtained contains the expected number of nodes but does not reach the expected number of edges. This first step returns a connected simple, acyclic graph, resulting in a topology similar to that of a tree. It represents the initial social links that will evolve during the simulation.

3) Adding random edges (connecting agents): The second phase allows us to link members of the crowd randomly. This step serves to reproduce the process of connecting people that know each other, either before joining the network or afterward. In order to implement different types of social links, we included a weighting strategy to the links. Each edge will be assigned a value that represents its type. We model three types of links: family, friendship link and professional.

4) Reinforcement of communities: This stage corresponds to the moment when the members of the network begin to establish relationships with other members who share the same friends. Two persons who didn't know each other beforehand can potentially become friends, which will lead to the creation of new links between them. The creation of edges according to this scheme allows the strengthening of communities, thereby increasing the agglomeration coefficient of the generated network. Unlike the first two phases that run before the launch of the simulation, the latter runs simultaneously with the mobility model.

\section{B. Adaptation of Susceptible, Infectious and Recovered (SIR) epidemic model for panic propagation}

The panic model was integrated into the mobility model and it uses the same environment and pedestrian modeling. The analogy between panic and contagion led us to a propagation model inspired by the epidemiological ones, and strengthened by the work of [12] and [3]. We adapted the SIR compartmental model [13] to describe the evolution and spread of panic within a crowd. Compartmental models are a technique used to simplify the mathematical modeling of infectious disease. For instance, the population is divided into compartments, with the assumption that all the individual within the same compartment share the same properties [13]. These models are usually modeled through ordinary differential equations (which are deterministic), but can also be viewed in a stochastic framework, which is as realistic as complicated to analyze (Fig 2a). Our version of the SIR model consists of four compartments where individuals are classified according to their emotional state:

- SP: compartment of people susceptible to panic.

- RL: compartment of people who behave with limited rationality. The individuals of this compartment have not yet reached the state of panic but are already disturbed.

- NR (P): compartment of people in a state of panic. The individuals of this compartment have reached the state of panic and behave irrationally.

- NP: compartment containing non-panicked people (calm). In fact, an individual is not assigned to the compartment NP until he reaches the emergency exit and leaves the simulation environment. Furthermore, we added an intermediate step before the transition to the state of panic, which is modeled via the RL compartment. It was introduced to express the non-spontaneous aspect of panic. This latter appears when the negative emotions of the individual (fear, stress) reach a predefined threshold. This last can be modeled by latency periods in the RL compartment that are randomly chosen to be able to express the heterogeneity among individuals. We also admit that some individuals have personal predispositions to panic. They will be in a panic state at the beginning of the simulation and will represent the initial panic transmission vector.

\section{1) Transitions between compartments:}

- Each susceptible individual moves from the compartment SP to the compartment RL with a transition rate $\left(\alpha_{1}\right)$ following direct contact with a panicked person.

- $\left(\alpha_{2}\right)$ represents the transition rate from the compartment RL to the compartment SP. This transition expresses the possibility that an individual RL can calm down after a certain period of time without reaching the state of panic.

- $\left(\beta_{1}\right)$ is the transition rate for which RL individuals transit to the compartment NR (P). In fact, each individual of the RL compartment spends some time in the latter. The choice of this parameter is very important because it allows changing phases in the process of panic propagation between an active phase, when panic spreads, and an inactive phase when it disappears.

- $\left(\beta_{2}\right)$ is the transition rate for which panicked people pass to the RL compartment. This transition happens for each individual after being panicked for a certain period of time. This aspect has been added to our model to take into account the ephemeral aspect of panic.

\section{Integration of the intermediate models}

In order to test and validate the proposed solutions, we integrated them in our mobility model and we adapt this latter to the modifications added. We start by integrating the social structure of the crowd obtained via the social model Small World in the decision making the process of agents. Afterward, we integrate panic model developed as an internal module in the mobility model.

1) Integration of the social structure into the decisionmaking process: Individuals will, now, adjust their displacement vector so as to evacuate while joining members that 
belong to their social group. This strategy was implemented using the distance maps obtained by the FMM method and provided by one of the two upper layers of the environment model in Fig 2b. Hence, each agent will have the possibility to identify the positions of individuals with whom they are socially linked and to choose the member to join according to the type of the link. The decision-making process consists of the following iterative steps:

1) Collect distance maps provided by the environment layers;

2) Collect the map of social links;

3) Identify the member to join among those having a social link with it;

4) Trigger a movement decision based on the collected data;

5) Perceive near environment information;

6) Adjust the decision according to the social forces model and behavior rules;

7) Take into consideration the individual properties that influence the decision;

8) Execute the decision.

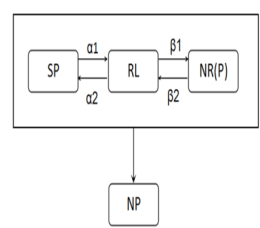

(a)

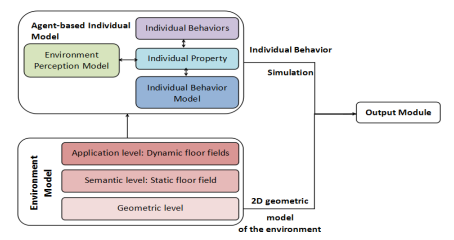

(b)
Fig. 2: (a) Proposed panic model, (b) Mobility model architecture.

2) Integration of panic model: The integration of panic model will change the system's flow. In fact, mobility and panic interact and influence each other mutually. Panic is nothing more than a structure contained in the crowd that will be activated when certain conditions are met. The general process of unfolding panic model follows two main phases. The first one takes place before the launch of the simulation. It allows the user to initialize the individuals initially panicked. Whereas, the second takes place during the simulation. In fact, it runs in parallel with the mobility model.

3) Panic consequences modeling: Once an individual becomes panicked, he behaves in a non-rational manner and adopts a social attitude toward the rest of the crowd, even toward members of their own social group. This behavioral disorder is reflected in our mobility model by the disappearance of social values and the emergence of violence and individualism. Violence was modeled by incrementing repulsive forces and individualism through the non-consideration of the social structure in the decision-making process. Nevertheless, panicked behaviors vary according to the nature of each person. We modeled this heterogeneity through different criteria and individual characteristics such as aggressiveness, restriction of the field of vision, increasing the speed of movement or reducing the comfort distance.

\section{RESUlts AND Discussion}

In this section, we study the flow while taking into account the social structure of the crowd and panic phenomenon. The validation of the mobility model was carried out on two steps: qualitative and quantitative. Several qualitative aspects of pedestrian dynamics were reproduced by our model such as lane formation, the arch phenomenon, oscillations at the bottleneck, etc (Fig 3a), and no more unrealistic congestions are encountered (Fig 3b). The quantitative study that we conducted regards pedestrians' flow through a narrow path (bottleneck) (Fig 3c). The experimental setup that we simulated was inspired by [14]. The results obtained using our mobility model correspond well to the real-world observations (Tab I, II).

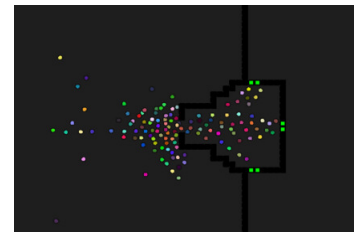

(a)

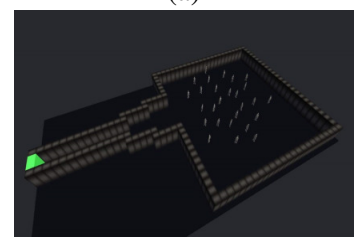

(c)

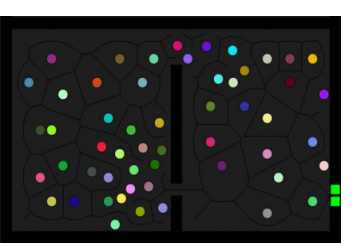

(b)

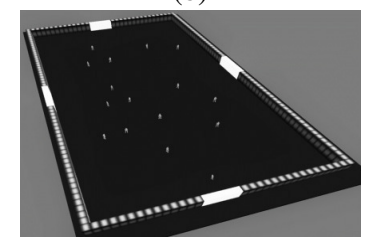

(d)
Fig. 3: (a) (b) Qualitative aspects obtained by the mobility model, (c) Bottleneck generation with our simulation framework, (d) Real simulation scenario

\section{A. Study of social structure impact}

First, we start with the simulation results obtained without introducing the social structure into the mobility model in

TABLE I: Experimental specific flow $J_{s, \exp }$ [14]

\begin{tabular}{|c|c|c|c|}
\hline $\mathrm{b}(\mathrm{m})$ & $N_{b}=20$ & $N_{b}=40$ & $N_{b}=60$ \\
\hline 0.8 & 1.86 & 1.77 & 1.61 \\
\hline 0.9 & 2.06 & 1.91 & 1.86 \\
\hline 1.0 & 2.19 & 2.08 & 1.9 \\
\hline 1.1 & 1.78 & 1.93 & 1.93 \\
\hline 1.2 & 2.31 & 1.81 & 1.97 \\
\hline
\end{tabular}

TABLE II: Values of the specific flow $J_{s}$ obtained using our Framework.

\begin{tabular}{|c|c|c|c|}
\hline $\mathrm{b}(\mathrm{m})$ & $N_{b}=20$ & $N_{b}=40$ & $N_{b}=60$ \\
\hline 0.8 & 1.73 & 1.45 & 1.40 \\
\hline 0.9 & 1.92 & 1.49 & 1.53 \\
\hline 1.0 & 2.07 & 1.63 & 1.66 \\
\hline 1.1 & 2.26 & 1.78 & 1.73 \\
\hline 1.2 & 2.35 & 1.83 & 1.86 \\
\hline
\end{tabular}


order to study the impact of this latter on the decision-making process without introducing panic. In order to distinguish social links influence, we run a series of simulations using the same scenario with the new and the old version of the mobility model. The test scenario we used is inspired by that illustrated in (Fig 3d).

Several qualitative aspects of pedestrian dynamics were reproduced by our model. Many of which were already obtained without the social aspect of the crowd such as, lane formation, the arch phenomenon, oscillations at the bottleneck. However, by introducing the social aspect congestion reappears, as well as the formation of reconciliation movements and training groups of different sizes, which is closer to reality.

\section{B. Study of panic propagation and its effects}

In order to simplify the study of panic propagation, we simulate without mobility. Since there is not a method to quantify the growth rates of emotion intensity [3], we vary the latency and infection period from one individual to another to express their emotional and psychological heterogeneity. Nevertheless, the proposed panic model is macroscopic, it describes the evolution of the population and not that of the individuals themselves. This evolution is considered continuous over time, which is reflected mathematically by an ordinary differential equation system :

$$
\left\{\begin{aligned}
\frac{d x}{d t} & =-\alpha_{1} \cdot x+\alpha_{2} \cdot y \\
\frac{d y}{d t} & =\alpha_{1} \cdot x-\left(\frac{\beta_{1}}{N} \cdot z+\alpha_{2}\right) \cdot y+\beta_{2} \cdot z \\
\frac{d z}{d t} & =\left(\frac{\beta_{1}}{N} \cdot y-\beta_{2}\right) \cdot z
\end{aligned}\right.
$$

The ratio $\frac{\beta_{1}}{N \cdot \beta_{2}} . y$ defines the basic reproduction number $R$. According to its value, panic will spread or shrink. Generally, it is the initial value $R_{0}$ that is used. We have either:

- $R_{0}=\frac{\beta_{1}}{\beta_{2}}>1$ : propagation,

- $R_{0}=\frac{\beta_{1}}{\beta_{2}}<1$ : shrinkage.

Several methods exist to solve the differential equations system. We used the approximation obtained by the classical Runge-Kutta and we implemented it then integrated it to our simulation platform. In order to validate the hypothesis about panic propagation, we conducted an experiment inspired by the one presented in [3]. We start with a population of 50 individuals susceptible to panic, 70 individuals of limited rationality and 5 panicked individuals. We vary the value of the transition rates $\beta_{1}$ and $\beta_{2}$ to verify the validity of the expression of the basic reproduction number. Fig $4 \mathrm{a}$ and $4 \mathrm{~b}$ show the evolution of the numbers of individuals in each compartment for $R_{0}$, respectively, greater than and less than 1.0 .

The obtained results show that the spread of panic and its shrinkage depend on the choice of the user. Moreover, the behavior of individuals can be defined according to their state. This aspect allows for implementing several cases in order to study and evaluate faithfully safety procedures. By introducing mobility, the social structure of the crowd evolves

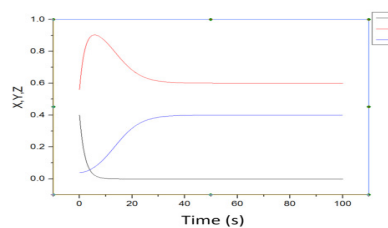

(a)

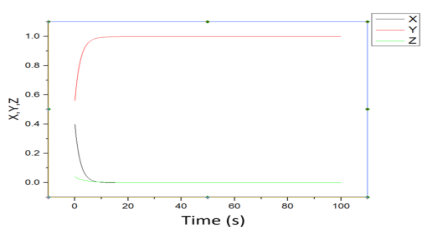

(b)
Fig. 4: Graphs showing the evolution of the number of SP, RL and $\mathrm{P}$ individuals for a basic reproduction number (a) $R_{0}>1$ and (b) $R_{0}<1$.

and promotes the spread of panic and allows it to reach isolated groups.

In order to position our model among the existing ones, we conducted a comparison between the models [3, 15, 16] and the proposed one according to the following criteria: reactive/cognitive approach, micro-level explicitness, macrolevel explicitness, communication forms, and panic behavior explicitness.

The reactive and cognitive approaches are related to how individuals are modeled in the system. Authors in [3, 15] adopted both the cognitive approach, while [16] and the proposed model combine both approaches.

The micro and macro levels parameters refer to the presence of components that represent the collectivity in an explicit fashion. Concerning the micro level explicitness: [16] and [3] deal with it using agent-based models, [15] on the other hand, uses collective agents and our model uses a hybridization of individual behavior model, social forces model, and global rules. The macro level explicitness, however, was addressed using: group formation with social forces by [16], imaginary and group mind formation by [15], without detail in [3] and using a Social crowd model in our solution.

The communication form parameter deals with the interactions among the collectivity of agents. These interactions can happen either directly, indirectly or through the process of perturbation and dissipation. The communication was of this latter type in [15] and, indirect in [16], and [3] as well as in the proposed model.

Finally, panic behavior parameter deals with the usage of the collective behavior formation stage. If such a stage is modeled and the transition is described in detail for each agent, they could behave more realistically, and the simulation could be closer to reality. This aspect was not available in [16], and was modeled using: a framework based on symbolic interactionism in [15], a macroscopic model for panic spread based on an epidemiological approach in [3] and in the proposed solution.

\section{CONCLUSION AND PERSPECTIVES}

In this paper, we developed a macroscopic panic model that permits to study the evolution of a panicked population. This model is based on an epidemiological approach and takes into account the social aspect of the crowd. We also integrated this model as an internal component of a microscopic mobility model to investigate and explore the complementarity between 
macroscopic and microscopic models and the possibility of enrichment between them. The obtained results are encouraging and replicate faithfully the real crowd evacuation situations.

As further work, we think there are topics that need to be deeply investigated in order to improve crowd simulation and analysis. First, human emotions represent one of the most important factors that affect the propagation of panic in the crowd. Thus, modeling emotions and their evolution process will systematically enhance the realism of panic propagation. Second, the role of an individual during the emergency situation is also one of the most important factors, which affect the process of decision making. Therefore, modeling the different roles that can be assigned to individuals and computing their influence on the crowd will be necessary for an even more realistic simulation. Third, human behavior depends on the state of each individual in the crowd, the latter can be obtained from sociological studies and empirical observations.

\section{REFERENCES}

[1] Anthony R Mawson. "Understanding mass panic and other collective responses to threat and disaster". In: Psychiatry: Interpersonal and biological processes 68.2 (2005), pp. 95-113.

[2] Tibor Bosse et al. "Modelling collective decision making in groups and crowds: Integrating social contagion and interacting emotions, beliefs and intentions". In: AUTON AGENT MULTI-AG 27.1 (2013), pp. 52-84.

[3] Haifa Abdelhak. "Modélisation des phénomènes de panique dans le cadre de la gestion de crise". $\mathrm{PhD}$ thesis. Université du Havre, 2013.

[4] Zhilu Yuan et al. "Simulation model of self-organizing pedestrian movement considering following behavior". In: Frontiers of Information Technology \& Electronic Engineering 18.8 (2017), pp. 1142-1150.

[5] Peng Lin, Jian Ma, and Siuming Lo. "Discrete element crowd model for pedestrian evacuation through an exit". In: Chinese Physics B 25.3 (2016), p. 034501.
[6] Mei Ling Chu et al. "Modeling social behaviors in an evacuation simulator". In: C. Animation and Virtual Worlds 25.3-4 (2014), pp. 373-382.

[7] Jinhuan Wang et al. "Modeling and simulating for congestion pedestrian evacuation with panic". In: Physica A: Statistical Mechanics and its Applications 428 (2015), pp. 396-409.

[8] Russell Hardin. Collective action. RFF Press, 2015.

[9] Lu Tan, Mingyuan $\mathrm{Hu}$, and Hui Lin. "Agent-based simulation of building evacuation: Combining human behavior with predictable spatial accessibility in a fire emergency". In: Inf. Sciences 295 (2015), pp. 53-66.

[10] Yan Li et al. "A grouping method based on grid density and relationship for crowd evacuation simulation". In: Physica A Stat. Mech. Appl. 473 (2017), pp. 319-336.

[11] Dirk Helbing and Peter Molnar. "Social force model for pedestrian dynamics". In: Physical review E 51.5 (1995), p. 4282.

[12] Farid Kadri, Babiga Birregah, and Eric Châtelet. "The impact of natural disasters on critical infrastructures: A domino effect-based study". In: J. of Homeland Security and Emergency Management 11.2 (2014), pp. 217-241.

[13] Fatima Zohra Younsi. "Mise ne palce d'un système d'aide à la décision pour le suivi et la prévention des épidémies". PhD thesis. Université d'Oran, 2016.

[14] Armin Seyfried et al. "New insights into pedestrian flow through bottlenecks". In: Transportation Science 43.3 (2009), pp. 395-406.

[15] Robson dos Santos França, Maria das Graças Bruno Marietto, and Margarethe Born Steinberger. "A Multiagent Model for Panic Behavior in Crowds”. In: (2009).

[16] Bachar Kabalan. "Crowd dynamics: modeling pedestrian movement and associated generated forces". PhD thesis. Université Paris-Est, 2016. 